\title{
Influência do tempo de síntese na obtenção de redes metal- orgânicas de cobre 2D via aquecimento hidrotérmico
}

\author{
Paulo Henrique Santos Oliveira ${ }^{1}$ (D), Alanis Joanna Silva Melo ${ }^{1}$, Arthur Felipe \\ de Farias Monteiro ${ }^{2}$ (D) \& Jarley Fagner Silva do Nascimento ${ }^{1}$
}

(1) Instituto Federal de Educação, Ciência e Tecnologia do Rio Grande do Norte - Campus Macau, Rua das Margaridas 300, COHAB 59500-000, Macau, Rio Grande do Norte, Brasil. E-mail: jarley.nascimento@ifrn.edu.br

(2) Universidade Federal de Pernambuco, Centro de Ciências Exatas e da Natureza, Departamento de Química Fundamental, Programa de Pós-Graduação em Química, Av. Prof. Moraes Rego 1235, Cidade Universitária 50670-901, Recife, Pernambuco, Brasil. E-mail: arthur.ffmonteiro@ufpe.br

Oliveira P.H.S., Melo A.J.S., Monteiro A.F.F. \& Nascimento J.F.S. (2020) Influência do tempo de síntese na obtenção de redes metal-orgânicas de cobre 2D via aquecimento hidrotérmico. Pesquisa e Ensino em Ciências Exatas e da Natureza, 4: e1538. http://dx.doi.org/10.29215/pecen.v4i0.1538

Editor acadêmico: Guilherme Leocárdio Lucena dos Santos. Recebido: 29 julho 2020. Aceito: 10 setembro 2020. Publicado: 01 novembro 2020.

Resumo: Neste trabalho, relatamos um estudo sobre a influência do tempo de síntese na obtenção do composto denominado bis(aqua)- $\mu$-[tereftalato- $\left.{ }_{k} \mathrm{O}\right]$ cobre (II) $\left(\mathrm{Cu}_{2}(\mathrm{BDC})\left(\mathrm{H}_{2} \mathrm{O}\right)_{2}\right)$, a partir da reação entre o sulfato de cobre penta-hidratado $\left(\mathrm{CuSO}_{4} \cdot 5 \mathrm{H}_{2} \mathrm{O}\right)$ e tereftalato de sódio $\left(\mathrm{Na}_{2} \mathrm{BDC}\right)$ em meio aquoso via método hidrotérmico convencional. O material já elucidado anteriormente foi obtido via difusão lenta em um tubo $\mathrm{H}$, e nessa ocasião foi obtido uma mistura de fases sendo uma delas a fase $\left(\mathrm{Cu}_{2}(\mathrm{BDC})\left(\mathrm{H}_{2} \mathrm{O}\right)_{2}\right)$. Observou-se que via método hidrotérmico, todas as amostras apresentam a presença do reagente precursor $\mathrm{CuSO}_{4} \cdot 5 \mathrm{H}_{2} \mathrm{O}$ não reagido identificado, a partir de seus picos característicos nos difratogramas. Avaliou-se dessa forma, que a partir de 24 horas de síntese se torna mais vantajoso o método descrito neste trabalho, pois será obtido um material com um maior grau de pureza da fase majoritária $\mathrm{Cu}_{2}(\mathrm{BDC})\left(\mathrm{H}_{2} \mathrm{O}\right)_{2}$, e menor presença do reagente precursor $\mathrm{CuSO}_{4} \cdot 5 \mathrm{H}_{2} \mathrm{O}$.

Palavras chave: Síntese, tempo, MOFs, tereftalatos de cobre.

Influence of synthesis time on obtainment of metal-organic networks of 2D copper via hydrothermal heating

Abstract: In this work, we report a study on the influence of the synthesis time in obtaining the compound called bis(aqua)- $\mu$-[terephthalate-kO] copper (II) $\left(\mathrm{Cu}_{2}(\mathrm{BDC})\left(\mathrm{H}_{2} \mathrm{O}\right)_{2}\right)$, from the reaction between copper sulfate pentahydrate $\left(\mathrm{CuSO}_{4} \cdot 5 \mathrm{H}_{2} \mathrm{O}\right)$ and sodium terephthalate $\left(\mathrm{Na}_{2} \mathrm{BDC}\right)$ in aqueous medium via conventional hydrothermal method. The material already elucidated was obtained via slow diffusion in an $\mathrm{H}$ tube, and on that occasion a mixture of phases was obtained, one of them being the phase $\left(\mathrm{Cu}_{2}(\mathrm{BDC})\left(\mathrm{H}_{2} \mathrm{O}\right)_{2}\right)$. It was observed that via the hydrothermal method, all samples have the presence of the precursor reagent $\mathrm{CuSO}_{4} .5 \mathrm{H}_{2} \mathrm{O}$ identified from their characteristic peaks in the diffractograms. It was evaluated in this way, that after 24 hours of synthesis, the method described in this work becomes more advantageous, since a material with a higher degree of purity of the majority phase $\mathrm{Cu}_{2}(\mathrm{BDC})\left(\mathrm{H}_{2} \mathrm{O}\right)_{2}$, and less presence of the reagent precursor CuSO4.5H2O.

Key words: Synthesis, time, MOFs, copper terephthalates.

\section{Introdução}

As redes metal-orgânicas (Metal-Organic Framework - MOFs) são compreendidas como uma ampla classe de materiais cristalinos com elevada porosidade, constituídos por íons 
metálicos ou clusters metálicos interconectados através de ligantes orgânicos (Barros et al. 2018). Tais estruturas, devido as suas características estruturais e topológicas, além das altas áreas de superfícies, apresentam poros ajustáveis, podendo se estender em mais de uma dimensão (Leite et al. 2017; Nascimento et al. 2017; Barros et al. 2018).

Uma sucessão de ligantes têm sido apresentados e empregados na síntese de novas MOFs, dentre eles, os policarboxilatos aromáticos são reservadamente interessantes devido a sua robustez química (Rodrigues et al. 2009). $\mathrm{O}$ ácido $1.4-\mathrm{H}_{2} \mathrm{BDC}$ exerce papel de destaque na construção de redes metalorgânicas, uma vez que pode atuar como ponte entre os centros metálicos e amparar abundantes modos de coordenação, dando origem a estruturas uni, bi ou tridimensionais (1D, 2D e 3D) (Lin et al. 2007).

A preparação de MOFs é significativamente afetada por variações sutis nas condições de reação como solvente, temperatura, proporção dos reagentes, concentração e tempo de síntese (Barros et al. 2018). O tempo de síntese é fator preponderante quando se trata da cristalinidade da rede. Avaliou-se nesse estudo, o tempo de síntese ideal para obtenção de um material com maior grau de pureza da estrutura cristalina $\mathrm{Cu}_{2}(\mathrm{BDC})\left(\mathrm{H}_{2} \mathrm{O}\right)_{2}$, a partir de método hidrotérmico convencional. Deakin et al. (1999) obtiveram a estrutura cristalina referida por método de difusão lenta em tubo $\mathrm{H}$ em vários dias, misturada a outra fase descrita pelos autores como, $\mathrm{Cu}_{2}(\mathrm{BDC})\left(\mathrm{H}_{2} \mathrm{O}\right)_{2} \cdot \mathrm{H}_{2} \mathrm{O}$, que apresentou propriedades magnéticas promissoras. Nessa ocasião, foi obtida uma mistura de fases, enquanto nesse trabalho, observou-se que a partir de 24 horas de síntese se torna mais vantajoso o método descrito, pois será obtido um material com um maior grau de pureza da fase majoritária $\mathrm{Cu}_{2}(\mathrm{BDC})\left(\mathrm{H}_{2} \mathrm{O}\right)_{2}$ e menor presença do reagente precursor $\mathrm{CuSO}_{4} \cdot 5 \mathrm{H}_{2} \mathrm{O}$ não reagido. Os materiais obtidos foram caracterizados por difração de Raios-X (DRX) e espectroscopia de infravermelho por transformada de Fourier (FTIR-ATR).

\section{Material e Métodos}

Todos os reagentes e solventes que foram utilizados não receberam purificação adicional. Ácido tereftálico (98\%) e sulfato de cobre penta hidratado $(98.9 \%)$ foram obtidos da Sigma-aldrich, e o Álcool Etílico (99.8\%) e hidróxido de sódio (97\%) foram obtidos da Vetec.

\section{Síntese da MOF}

Utilizou-se as mesmas razões estequiométricas dos reagentes precursores, na síntese proposta por Deakin et al. (1999). Foram adicionados a um béquer contendo $10 \mathrm{ml}$ de água destilada, $0.0735 \mathrm{mmol}$ de ácido tereftálico e observou-se a turbidez do sistema devido à baixa solubilidade do ligante em água. $\mathrm{O}$ sistema foi mantido sob agitação constante e foram adicionados $0.1470 \mathrm{mmol}$ de $\mathrm{NaOH}$, para obtenção do tereftalato de sódio que é solúvel em meio aquoso. Em seguida, adicionou-se ao mesmo béquer $0.075 \mathrm{mmol}$ de $\mathrm{CuSO}_{4} \cdot 5 \mathrm{H}_{2} \mathrm{O}$, e o sistema foi mantido sob agitação durante 20 minutos até a homogeneização do sistema. Em seguida, a solução resultante foi transferida para um reator de teflon com capacidade de $20 \mathrm{ml}$ com "camisa" de aço, e levado para uma estufa a $120^{\circ} \mathrm{C}$, em tempos diferentes de reação $(6 \mathrm{~h}, 12 \mathrm{~h}, 24 \mathrm{~h}$ e 48h), com o intuito de verificar qual seria o tempo ideal para formação da MOF sob essas condições, uma vez que já foi realizada esta síntese por difusão lenta num tubo $\mathrm{H}$ (Deakin et al. 1999), enquanto estamos propondo uma síntese hidrotérmica por aquecimento convencional. Os cristais obtidos foram lavados em triplicata com etanol e centrifugados por 60 segundos a 3600 rpm. Os pós-obtidos foram secos por 24 horas na estufa a $70^{\circ} \mathrm{C}$ e posteriormente caracterizados.

\section{Caracterização da MOF}

As medidas de difração de raios-X, foram feitas em um difratômetro Bruker D2 Phaser, utilizando radiação $\operatorname{Cuk} \alpha(\lambda=1.5406 \AA \hat{)})$ contendo filtro de níquel. A faixa de obtenção do difratograma foi entre $3^{\circ}$ e $70^{\circ}$, com passo de $0.02^{\circ}$, sendo 10 passos por segundo. A estrutura 2D proposta $\mathrm{Cu}(\mathrm{BDC})\left(\mathrm{H}_{2} \mathrm{O}\right)_{2}$ para as amostras, foi obtida a partir do programa Diamond da crystal impact a partir da ficha catalográfica do CCDC 141928 (The Cambridge Crystallographic Data 
Centre -CCDC) (Allen et al. 1979). Os espectros de infravermelho por transformada de Fourier (FTIR-ATR) foi obtido com o espectrômetro Bruker FTIR - Vertex 70, com platinun ATR, analisando o pó do material em uma faixa entre $\left.4000-400 \mathrm{~cm}^{-1}\right)$.

\section{Resultados e Discussão}

A estrutura cristalina e as fases das amostras obtidas foram caracterizadas via difração de raios-X (DRX). As amostras tiveram seus padrões de difração comparadas com a fase do ligante puro e do padrão simulado referente a ficha CCDC 141928 (The Cambridge Crystallographic Data Centre - CCDC) (Allen et al. 1979), relatada em trabalho anterior (Deakin et al. 1999). O padrão simulado refere-se a estrutura $\mathrm{Cu}_{2}(\mathrm{BDC})\left(\mathrm{H}_{2} \mathrm{O}\right)_{2}$, como pode ser visto na Figura 1.

Na Figura 1, ao comparar os padrões de difração das amostras ao do ligante livre (1.4$\mathrm{H}_{2} \mathrm{BDC}$ ), verificou-se um perfil completamente distinto dos difratogramas das amostras quando comparado a fase do ligante puro, o que sugere que a síntese foi satisfatória. A variação do tempo de síntese apresentou influência na formação da estrutura, uma vez que os picos em $29.2^{\circ}$, $31.8^{\circ}, 35.2^{\circ}$ e $38.7^{\circ}$ são observados em maior intensidade para as amostras obtidas em tempos menores de síntese (6h e $12 \mathrm{~h}$ ). Realizou-se a comparação a ficha do ICCD (International Center for Diffraction Data), ref. $\mathrm{N}^{\circ}$ 9-348, e estes picos são característicos do sulfato de cobre pentahidratado $\left(\mathrm{CuSO}_{4} .5 \mathrm{H}_{2} \mathrm{O}\right)$, mostrando que parte desse reagente precursor não reagiu. As amostras sintetizadas em 24 e 48 horas de síntese (Cu(BDC)_HID24H e $\mathrm{Cu}(\mathrm{BDC})_{-} H \mathrm{HID} 48 \mathrm{H}$, respectivamente, apresentam difratogramas consistentes com a fase simulada $\mathrm{Cu}_{2}(\mathrm{BDC})\left(\mathrm{H}_{2} \mathrm{O}\right)_{2}$, identificado principalmente pelo pico mais intenso em $8.1^{\circ}$, e os picos em $12.4^{\circ}, 15.1^{\circ}$ e $16.3^{\circ}$, apresentando menor intensidade dos picos característicos oriundos do $\mathrm{CuSO}_{4} .5 \mathrm{H}_{2} \mathrm{O}$, o que demonstra que a partir de 24 horas de síntese teríamos a formação de uma estrutura cristalina com maior rendimento de formação do produto majoritário, $\mathrm{Cu}_{2}(\mathrm{BDC})\left(\mathrm{H}_{2} \mathrm{O}\right)_{2}$.

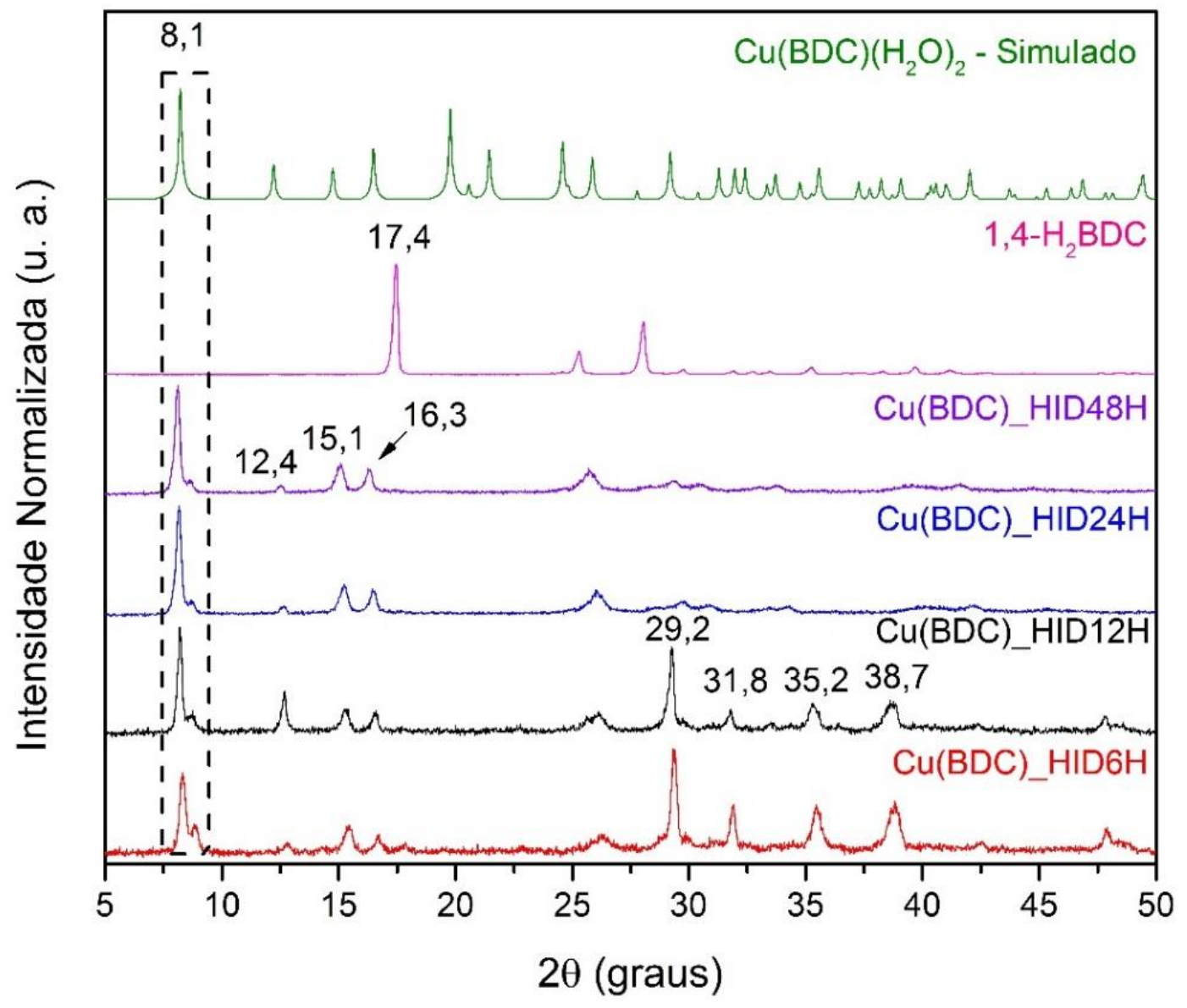

Figura 1. Difração de raios-X das amostras sintetizadas em $6 \mathrm{~h}, 12 \mathrm{~h}, 24 \mathrm{~h}$ e $48 \mathrm{~h}$, comparadas a fase do ligante puro (1.4- $\mathrm{H}_{2} \mathrm{BDC}$ ) e do padrão simulado. 
Com base nos padrões de DRX das amostras e utilizando o software Diamond (Pennington 1999), obteve-se a estrutura cristalina da fase majoritária identificada em todas as amostras sintetizadas. A estrutura é uma rede metalorgânica 2D, que apresenta os cobres inseridos em uma geometria quadrado-piramidal distorcido como pode ser visto na Figura 2. Os centros metálicos de $\mathrm{Cu}^{2+}$ possuem quatro ligações, duas com o oxigênio das moléculas de água nas posições apicais, e duas pelo oxigênio do ligante tereftalato (1.4-BDC) ligado em pontes no modo cis-bi-monodentado, originando uma sobreposição de camadas (Figura 2). Convencionalmente, o modo bi-monodentado do ligante 1.4-BDC, origina pontes trans-ligadas (Deakin et al. 1999). A conformação cis, é possivelmente estabilizada a partir da ligação de hidrogênio entre, o grupo CO livre do ligante e a água coordenada do complexo vizinho ao longo da cadeia (linha tracejada preta) (Deakin et al. 1999).

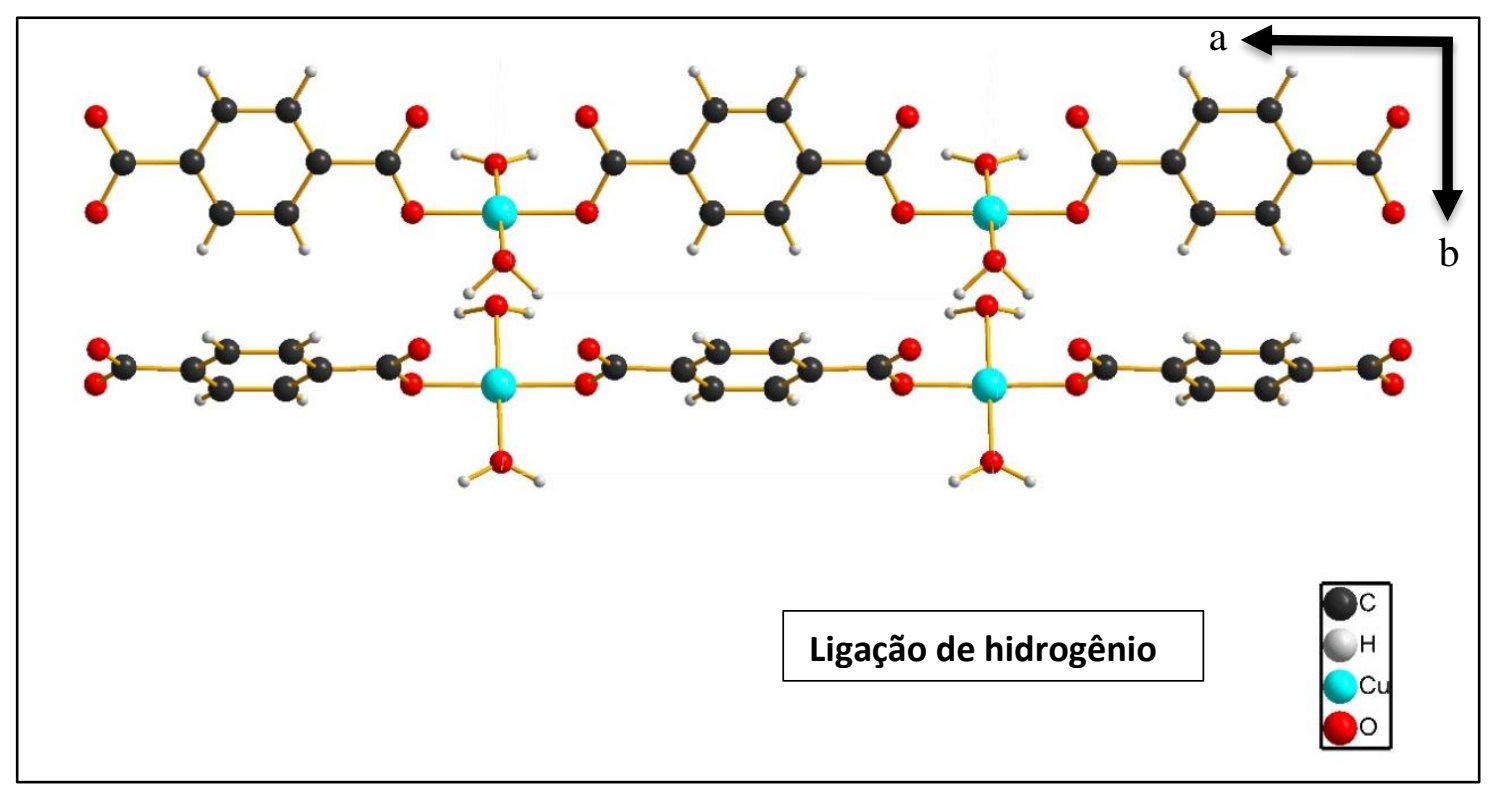

Figura 2. Projeção da estrutura 2D parcialmente expandida ao longo dos eixos cristalográficos a e c. código de cores: preto (carbono), branco (hidrogênio), azul (cobre) e vermelho (oxigênio).

As amostras preparadas em diferentes tempos de síntese via método hidrotérmico, foram caracterizadas por espectroscopia de infravermelho com transformada de Fourier (FTIRATR), visando avaliar os principais modos de coordenação, que estão apresentados na Figura 3. Ao comparar os espectros do ligante livre $\left(1.4-\mathrm{H}_{2} \mathrm{BDC}\right)$ com os das amostras preparadas, observa-se a ausência da banda do ligante em $1671 \mathrm{~cm}^{-1}$, que está atribuída a vibração de estiramento da ligação $\mathrm{C}=\mathrm{O}$ no grupo $\mathrm{COOH}$, o que demonstra a coordenação dos centros metálicos pelos grupos carboxilatos, e que não houve excesso de ligante após a reação. Observase para as amostras $\mathrm{Cu}(\mathrm{BDC}) \_$HID6H e $\mathrm{Cu}(\mathrm{BDC})$ HID12H de forma mais clara, uma banda na região de $1576 \mathrm{~cm}^{-1}$ e duas bandas em torno de 1389 e $1346 \mathrm{~cm}^{-1}$, que estão relacionadas respectivamente, aos estiramentos assimétricos $\left(v_{\text {as }}\right)$ e simétricos $\left(v_{\mathrm{s}}\right)$ correspondente aos grupos COO $^{-}$(Deacon \& Phillips 1980). O surgimento de duas bandas de $v_{\mathrm{s}}$, está presente em todas as amostras, o que confirma a presença de duas fases para as mesmas, corroborando os resultados de DRX. As diferenças entre o $\left(v_{\text {as }}\right)$ e $\left(v_{\mathrm{s}}\right)$ são respectivamente $203 \mathrm{~cm}^{-1}$ e $236 \mathrm{~cm}^{-1}$ o que indica modos de coordenação no modo monodentado para ambas as fases. A diferença entre o $\left(v_{\mathrm{as}}\right)$ e $\left(v_{\mathrm{s}}\right)$ para a MOF $\mathrm{Cu}_{2}(\mathrm{BDC})\left(\mathrm{H}_{2} \mathrm{O}\right)_{2}$, indica a coordenação no modo monodentado (Deacon \& Phillips 1980), consistente com a estrutura proposta na Figura 2. A absorção em $3574 \mathrm{~cm}^{-1}$, está relacionada com a coordenação de moléculas de água na estrutura das MOFs, o que também está em consonância com a estrutura proposta na Figura 2. As absorções que ocorrem na faixa de 1183-908 $\mathrm{cm}^{-1}$ e de 796-654 cm $\mathrm{cm}^{-1}$, estão relacionadas com as vibrações das ligações C-H do anel benzênico do ligante, no plano e fora do plano respectivamente, sendo observadas em todos os espectros mesmo após a coordenação do centro metálico (Deacon \& Phillips 1980). 


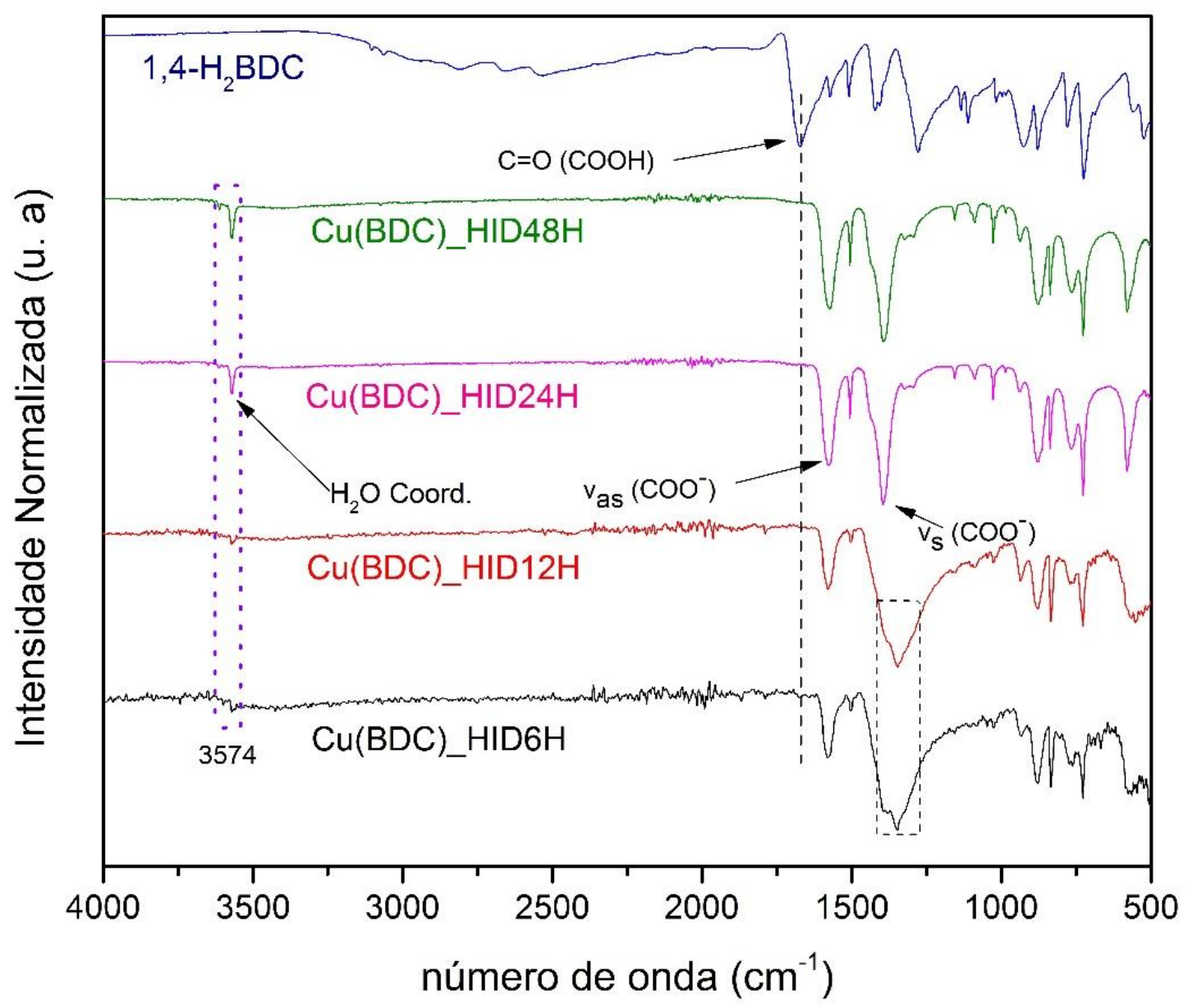

Figura 3. Espectros de FTIR-ATR das amostras sintetizadas em $6 \mathrm{~h}, 12 \mathrm{~h}, 24 \mathrm{~h}$ e $48 \mathrm{~h}$, comparados ao espectro do ligante puro (1.4- $\left.\mathrm{H}_{2} \mathrm{BDC}\right)$.

\section{Conclusões}

A síntese das redes metalorgânicas de cobre utilizando apenas água como solvente via método hidrotérmico, se mostrou eficaz e foi confirmada a partir das análises de DRX e FTIRATR. No entanto, as amostras obtidas, apesar de ter uma fase majoritária correspondente a estrutura simulada $\mathrm{Cu}(\mathrm{BDC})\left(\mathrm{H}_{2} \mathrm{O}\right)_{2}$, já elucidada (Deakin et al. 1999), apresentaram $\mathrm{CuSO}_{4} \cdot 5 \mathrm{H}_{2} \mathrm{O}$ não reagido. $\mathrm{O}$ tempo de síntese mostrou forte influência na obtenção das MOFs, uma vez que os picos de difração característicos do $\mathrm{CuSO}_{4} .5 \mathrm{H}_{2} \mathrm{O}$ não reagido, foram mais intensos para as duas amostras com menor tempo de síntese $6 \mathrm{~h}$ e $12 \mathrm{~h}$. A estrutura cristalográfica proposta para a MOF, está consistente com as bandas do espectro de infravermelho observadas para as amostras. Com base no que foi supracitado, podemos inferir, que o tempo ideal para obter materiais com maior grau de pureza não deve ser menor que o de 24 horas.

\section{Referências}

Allen F.H., Bellard S., Brice M.D., Cartwright B.A., Doubleday A., Higgs H., Hummelink T., Hummelink-Peters B.G., Kennard O., Motherwell W.D.S., Rodgers J.R. \& Watson D.G. (1979) The Cambridge Crystallographic Data Centre: computer-based search, retrieval, analysis and display of information. Acta Crystallographica Section B: Structural Crystallography and Crystal Chemistry, 35(10): 2331-2339. https://doi.org/10.1107/S0567740879009249

Barros B.S., de Lima Neto O.J., de Oliveira Frós A.C. \& Kulesza J. (2018) Metal-Organic Framework Nanocrystals. ChemistrySelect, 3(26): 7459-7471.

https://doi.org/10.1002/slct.201801423 
Deacon G.B. \& Phillips R.J. (1980) Relationships between the carbon-oxygen stretching frequencies of carboxylato complexes and the type of carboxylate coordination. Coordination Chemistry Reviews, 33(3): 227-250. https://doi.org/10.1016/S0010-8545(00)80455-5

Deakin L., Arif A.M. \& Miller J.S. (1999) Observation of Ferromagnetic and Antiferromagnetic Coupling in 1-D and 2-D Extended Structures of Copper(II) Terephthalates. Inorganic chemistry, 38(22): 5072-5077. https://doi.org/10.1021/ic990400r

Leite A.K.P., Barros B.S., Kulesza J., Nascimento J.F.S.D., Melo D.M.D.A. \& Oliveira A.A.S.D. (2017) Modulator Effect of Acetic Acid on the Morphology of Luminescent Mixed LanthanideOrganic Frameworks. Materials Research, 20(supl. 2): 681-687. http://dx.doi.org/10.1590/19805373-mr-2016-1015

Lin Z., Wragg D.S., Warren J.E. \& Morris R.E. (2007) Anion Control in the Ionothermal Synthesis of Coordination Polymers. Journal of the American Chemical Society, 129(34): 10334-10335. https://doi.org/10.1021/ja0737671

Nascimento J.F.S., Barros B.S., Kulesza J., de Oliveira J.B.L., Leite A.K.P. \& de Oliveira R.S. (2017) Influence of synthesis time on the microstructure and photophysical properties of Gd-MOFs doped with $\mathrm{Eu}^{3+}$. Materials Chemistry and Physics, 190: 166-174. https://doi.org/10.1016/j.matchemphys.2017.01.024

Pennington W.T. (1999) DIAMOND - Visual Crystal Structure Information System. Journal of Applied Crystallography, 32(5): 1028-1029. https://doi.org/10.1107/S0021889899011486

Rodrigues M.O., Paz F.A.A., Freire R.O., de Sá G.F., Galembeck A., Montenegro M.C., Araújo A.N. \& Alves Jr.S. (2009) Modeling, structural, and spectroscopic studies of lanthanide-organic frameworks. The Journal of Physical Chemistry B, 113(36): 12181-12188.

https://doi.org/10.1021/jp9022629 